\title{
TAGUNG
}

\section{Die Europäische Union in unruhigem Fahrwasser: Eine deutsch-nordisch-baltische Reformpartnerschaft in Zeiten innen- und außenpolitischer Herausforderungen}

\author{
Christian Opitz*
}

Die Europäische Union erlebt unruhige Zeiten. Selten wie nie zuvor bestimmen innenwie außenpolitische Herausforderungen die europäischen Denk- und Entscheidungsprozesse. Die Wahlen zum Europäischen Parlament im Mai 2014 läuteten einen neuen Abschnitt ein, der sich unter den gegenwärtigen Vorzeichen als richtungsgebend für die $\mathrm{Zu}$ kunft herausstellen könnte. Wichtige Personal- und Prioritätsentscheidungen sind derzeit in der Diskussion, die die inhaltliche Ausgestaltung der Europäischen Union in den nächsten Jahren mit Leben füllen sollen. Die langsame aber stetige wirtschaftliche Erholung könnte zu der oft (herbei-)gewünschten Aufbruchsstimmung beitragen. Die Krise in der Ukraine und der Konflikt mit Russland dürften diese hingegen wieder bremsen. Alle Seiten betonen, nicht an einer Neuauflage des Kalten Krieges interessiert zu sein, aber Rhetorik und Handlungen zeugen von einem längst überwunden geglaubten Misstrauen. Die Europäische Union steht vor ihrer möglicherweisen größten außenpolitischen Herausforderung in den letzten Jahrzehnten zu einem Zeitpunkt, zu dem die innere Verfasstheit der Union ihre Handlungsfähigkeit immer wieder infrage stellt.

In solchen ungewissen Zeiten erlangen Gesprächskreise wie das Deutsch-Nordisch-Baltische Forum (DNBF) einen besonderen Stellenwert. Unter der Überschrift „Upgrading the German-Nordic-Baltic Partnership in the EU: Common Values, Mutual Interests and New Challenges" versammelten sich mehr als

\section{$6^{\text {th }}$ German Nordic Baltic Forum 2014. Upgrading the German-Nordic- Baltic Partnership in the EU: Common Values, Mutual Interests and New Challenges}

Tagung des Latvian Institute of International Affairs und des Instituts für Europäische Politik (IEP)

Mit freundlicher Unterstützung des Auswärtigen Amtes, Berlin, und der Botschaft der Bundesrepublik Deutschland, Riga.

7./8. Juli 2014, Riga

\section{Welcome}

Dr. Andris SPRŪDS, Director, Latvian Institute of International Affairs (LIIA), Riga

Prof. Dr. Mathias JOPP, Director, Institut für Europäische Politik (IEP), Berlin

Reinventing the Eastern Partnership: Opportunities and Constraints

Chair: Juris POIKĀNS, Ambassador at Large (Eastern Partnership), Ministry of Foreign Affairs of the Republic of Latvia, Riga

Keynotes:

Joachim BLEICKER, Director for Basic Issues of EU External Relations and Relations with EU Member States, Federal Foreign Office, Berlin

Ojārs ĒRIKS KALNIN̦Š, Chairman, Foreign Affairs Committee of the Parliament of the Republic of Latvia, Riga

* Christian Opitz, MSSc., Forschungsassistent, Stiftung Wissenschaft und Politik, Berlin. 
60 Teilnehmer im lettischen Riga zum sechsten Treffen des DNBF. Die Konferenz bot akademischen Experten sowie Verantwortlichen aus den Ministerien und Parlamenten der teilnehmenden Länder die Gelegenheit zum Meinungsaustausch und zur Diskussion gemeinsamer Handlungsstrategien in einer anregend informellen Atmosphäre.

Der deutsche Staatsminister für Europa im Auswärtigen Amt Michael Roth steckte in seiner Keynote das Terrain der folgenden Diskussionen ab. Aus seiner Sicht werden fünf Bereiche für die kurz- bis mittelfristige Zukunft der Europäischen Union entscheidend sein: eine gestärkte Wirtschafts- und Währungsunion, ein umfassenderer Ansatz in der Sozialpolitik, eine sichere und nachhaltige Energieversorgung, der Schutz der Grundwerte sowie eine zielführende Nachbarschaftspolitik. Diese Prioritäten wurden von den Teilnehmern der vier Paneldiskussionen jeweils aus unterschiedlichen Blickwinkeln aufgegriffen und intensiv erörtert. Anstatt die Diskussionen im Detail wiederzugeben soll sich hier auf die überspannenden Grundthemen fokussiert werden, die sich wie ein roter Faden durch das zweitägige Forum zogen: die Bedeutung von interner und externer Kommunikation für die Europäische Union, die Weiterentwicklung von existierenden EUStrategien, und die Frage der Führungsrolle seitens der nordischen, baltischen und deutschen Verantwortlichen bei notwendigen Reformen im europäischen Kontext.

\section{Eine bessere interne und externe Kommuni- kation}

Themenübergreifend unterstrichen die Diskussionen immer wieder die zentrale Rolle der Kommunikation in der und für die EUPolitik. Mehrere Redner wiesen in diesem Zusammenhang darauf hin, dass Kommunikationsinhalte und -ziele eine interne wie externe Dimension besitzen. Innerhalb der Europäischen Union sind fortwährende Diskussionen vonnöten, die die politische, wirtschaftliche und soziale Basis des Gemein-
Panelists:

Dr. Katrin BÖTTGER, Deputy Director, Institut für Europäische Politik (IEP), Berlin

Dr. Sinikukka SAARI, Senior Researcher, Finnish Institute of International Affairs (FIIA), Helsinki

Dr. Iris KEMPE, Senior Adviser for Culture and Education, Council of the Baltic Sea States, Stockholm

Reviewing Europe 2020 Strategy: Making Stabilization Sustainable

Chair: Prof. Dr. Ramūnas VILPIŠAUSKAS, Institute of International Relations and Political Science, Vilnius University, Vilnius

\section{Panelists:}

Dr. Tuulia HAKOLA, Director, Structural Policy Unit, Ministry of Finance of Finland, Helsinki

Jo LEINEN, MEP, President of the European Movement International (EMI), European Parliament, Brussels

Märt LOITE, Deputy Strategy Director, Government Office of Estonia, Tallinn

Aldis AUSTERS, Researcher, Latvian Institute of International Affairs (LIIA), Riga

Prof. Dr. Ansgar BELKE, Chair of Macroeconomics, University of Duisburg-Essen

Key note addresses: German-Nordic-Baltic Partnership: Challenges, Interests, Values

Andrejs PILDEGOVIČS, State Secretary, Ministry of Foreign Affairs of the Republic of Latvia, Riga

Michael ROTH, MP, Minister of State for Europe, Federal Foreign Office, Berlin

\section{The EU as a Foreign Player: Interests and} Instruments

Chair: Dr. Gunilla HEROLF, Fellow, Royal Swedish Academy of War Sciences (RSAWS), Stockholm

Panelists:

H.E. Ričardas DEGUTIS, Ambassador of the Republic of Lithuania to Latvia, Riga

Marko MIHKELSON, Chairman, Foreign Affairs Committee, Estonian Parliament, Tallinn

Carl HARTZELL, Senior Policy Adviser, Strategic Planning Division, European External Action Service, Brussels

Niklas HELWIG, Research Fellow, Finnish Institute of International Affairs (FIIA), Helsinki 
schaftsprojekts und seine möglichen $\mathrm{Zu}$ kunftspfade ausloten. Zur gleichen Zeit wird die Vermittlung von Inhalt und Form der außenpolitischen Ziele der Europäischen Union immer wichtiger. Auf Seiten externer Akteure könnte somit ein besseres Verständnis für oder zumindest Klarheit über die Intentionen der Europäischen Union hergestellt werden.

Der Aufstieg von EU-skeptischen und rechtspopulistischen Parteien im Zuge der Wahlen zum Europäischen Parlament im Mai 2014 diente als prominentes Beispiel, welches die Notwendigkeit für eine realitäts- und bürgernahe Kommunikation aufzeigt. Die wachsende Verbreitung und Akzeptanz von Parolen gegen die Europäische Union und ihre zentralen Werte verdeutlichen in erster Linie eine tiefgreifende Verunsicherung und mangelnde Kenntnis von Sinn und Zweck des Gemeinschaftsprojekts innerhalb der Bevölkerung des Kontinents. Im Kontext der politischen Verantwortung für wirtschaftliche Verfehlungen seitens der Nationalstaaten, scheint es den Befürwortern immer schwerer zu fallen, die Erfolge der Europäischen Union zu vermitteln., Alte' Argumente wie jene von europäischer Versöhnung und Frieden - so wichtig diese auch heute noch sind - müssen von einem Diskurs flankiert und verstärkt werden, der die direkten Vorteile für jede Bürgerin und jeden Bürger in der Gegenwart und $\mathrm{Zu}-$ kunft verdeutlicht. Abgesehen von potenziell notwendigen Veränderungen im institutionellen Gefüge, ist eine solche selbstbewusste Binnenkommunikation, welche die EU-Bevölkerung von der fortwährenden Relevanz des Gemeinschaftsprojekts überzeugt, eine der größten Herausforderungen.

Viele Redebeiträge verwiesen darüber hinaus auf die wachsende Notwendigkeit, die außenpolitischen Ziele der Europäischen Union klar und plausibel gegenüber anderen Akteuren darzustellen. Die Östliche Partnerschaft veranschaulicht hierbei, was passieren kann,
EU Institutional Challenges after the European Parliament Elections of 2014

Chair: Kārlis BUKOVSKIS, Deputy Director, Latvian Institute of International Affairs (LIIA), Riga

Panelists:

Prof. Dr. Ahto LOBJAKAS, Researcher, Estonian Foreign Policy Institute (EVI), Tallinn

Kristīne NAŠENIECE, Director, EU Coordination Department, Ministry of Foreign Affairs of the Republic of Latvia, Riga

Prof. Dr. Rudolf HRBEK, Institute for Political Science, University of Tübingen

Dr. Zanda KALNIN̦A-LUKAŠEVICA, Chairwoman, European Affairs Committee of the Parliament of the Republic of Latvia, Riga

\section{Concluding Remarks}

Dr. Ilze RŪSE, Director, European Department, Ministry of Foreign Affairs of the Republic of Latvia, Riga

Dr. Funda TEKIN, Research Fellow, Institut für Europäische Politik (IEP), Berlin

wenn die Europäische Union ihre Strategie und Vorhaben gegenüber der anderen Seite nicht verständlich machen kann. Dies hatte im konkreten Fall gravierende Auswirkungen. Auf der einen Seite sahen sich die Regierungen und Gesellschaften in den Ländern der Östlichen Partnerschaft ${ }^{1}$ vor die schwerwiegende Entscheidung gestellt: entweder mit den Assoziierungsabkommen eine definitive Hinwendung zur Europäischen Union zu vollziehen bei gleichzeitiger Abkehr von historischen Einbettungen oder spiegelbildlich den Schritt mit umgekehrten Vorzeichen durchzuführen. Diese innerhalb der Ukraine unterschiedlich interpretierte Schicksalsfrage trug maßgeblich zu den wachsenden Spannungen zwischen verschiedenen Bevölkerungsgruppen sowie $\mathrm{zu}$ dem bewaffneten Konflikt in der Ukraine bei.

Russland, auf der anderen Seite, fühlte sich von den Entwicklungen in seiner Nachbarschaft bedroht und ergriff unerwartet drasti-

1 Die Östliche Partnerschaft umfasst die sechs ehemaligen Sowjetrepubliken Armenien, Aserbaidschan, Georgien, Moldawien, Ukraine und Weißrussland. 
sche Maßnahmen. Unabhängig davon, wie man die russische Aktionen bewertet - die Konferenzteilnehmer waren sich in der Verurteilung der Regierung Putins einig - haben die Geschehnisse rund um die Ukraine doch auch verdeutlicht, dass die Europäische Union ihre Politik nach außen deutlicher kommunizieren muss. Gegenseitiges Verständnis setzt klare und offene Kommunikation voraus. Dies könnte nicht nur zu einer Angleichung der Denkweisen der Regierungen in den EU-Mitgliedstaaten auf der einen und denen in den Ländern der Östlichen Partnerschaft und Russland auf der anderen Seite beitragen. In Anbetracht des eingeschränkten Zugangs zu unabhängigen Medien sollte die Europäische Union auch versuchen, die dortigen Gesellschaften besser über ihre Sichtweisen und Pläne zu informieren. Mehrere Experten drängten auf eine verstärkt strategische Dimension der Östlichen Partnerschaft. Es muss demzufolge klarer kommuniziert werden, was die Europäische Union durch ihre Maßnahmen in der östlichen Nachbarschaft erreichen möchte, und was nicht.

\section{Bestehende Strategien nachjustieren}

Eine klare und offene Kommunikation von Intentionen setzt gewöhnlich eine Formulierung von eigenen spezifischen Strategien voraus. Während die Europäische Union eine Vielzahl an Agenden und Programmen betreibt, hoben mehrere Diskussionsbeiträge die Notwendigkeit hervor, diese Aktivitäten fortwährend nach zu justieren. Es war demnach Konsens, dass veränderte Umstände zu einer Adaptierung und Spezifizierung führen sollten. Eine große Herausforderung in diesem Zusammenhang besteht jedoch in der Tatsache, dass ein Kurs, der nach zeit- und ressourcenaufwendigen Verhandlungen zwischen 28 Staaten eingeschlagen wurde, wenig Aussicht auf eine flexible Neuausrichtung be- sitzt. Trotzdem können Strategien nur dann effektiv sein und ihren zugrundeliegenden Zielen dienen, wenn ihnen ein Mindestmaß an Anpassungsfähigkeit im Lichte gegenwärtiger Entwicklungen zugestanden wird.

Die „Europa 2020“-Strategie ${ }^{2}$ illustriert die Notwendigkeit zur fortlaufenden Aktualisierung von existierenden Programmen. Die auf zehn Jahre angelegte Strategie aus dem Jahr 2010 verfolgt das Ziel eines intelligenten, nachhaltigen und integrativen Wirtschaftswachstums. Insbesondere die Koordination zwischen nationalen und europäischen Initiativen soll verbessert werden. Als Reaktion auf die globale Finanzkrise lanciert, ist die Strategie vorrangig darauf ausgelegt, das Wachstums- und Produktivitätsniveau auf dem Kontinent zu steigern. Seit 2010 haben sich die Volkswirtschaften in den Mitgliedstaaten jedoch zum Teil sehr unterschiedlich entwickelt. Ein Experte verdeutlichte hierbei die Situation in Lettland, wo die anhaltende Emigration der jungen und gut-qualifizierten Generation eines der Hauptprobleme bildet. Es wird demnach zunehmend wichtig, die gemeinschaftlichen EU-Ziele mit den divergierenden wirtschaftlichen und sozialen Gegebenheiten auf der nationalen Ebene in Einklang zu bringen. Die kürzlich veröffentlichte Bestandsaufnahme der „Europa 2020“-Strategie $^{3}$ seitens der Kommission ist ein wichtiger erster Schritt, der jedoch von zusätzlichen Maßnahmen flankiert werden muss.

Die Östliche Partnerschaft ist ein weiteres Beispiel für eine bestehende Strategie, die veränderte Realitäten berücksichtigen muss, will sie auch weiterhin zielführend sein. Viele der Teilnehmer sahen in der Östlichen Partnerschaft eine Erfolgsgeschichte seit ihrer Verabschiedung 2009. Allerdings war es bereits vor dem Vilnius-Gipfel im November 2013 und den nachfolgenden Entwicklungen

2 Europäische Kommission: Mitteilung der Kommission. Europa 2020. Eine Strategie für intelligentes, nachhaltiges und integratives Wachstum, KOM (2010) 2020.

3 Europäische Kommission: Mitteilung der Kommission an das Europäische Parlament, den Rat, den Europäischen Wirtschafts- und Sozialausschuss und den Ausschuss der Regionen. Bestandsaufnahme der Strategie Europa 2020 für intelligentes, nachhaltiges und integratives Wachstum, COM (2014) 130. 
offensichtlich, dass die Strategie einer Reform bedurfte. Die Krise in der Ukraine hat eine beträchtliche Dynamik entfacht und beschleunigte entsprechende Reformüberlegungen. Sie unterstrich auch und vor allem die Notwendigkeit zur Erklärung und Differenzierung der europäischen Politik. Zunächst muss die Europäische Union wiederum klar kommunizieren, dass die Östliche Partnerschaft kein Automatismus zur Aufnahme in die Gemeinschaft ist. Mehrfach wurde von verschiedenen Rednern betont, dass eine diesbezügliche Erweiterung mittel- bis langfristig nicht erfolgen könne und solle. Vonnöten ist hingegen ein maßgeschneidertes Vorgehen, welches auf die sechs Länder und deren individuelle Situation und Reformfortschritte eingeht. Ein Diplomat betonte in diesem Zusammenhang ausdrücklich, dass diese Differenzierung nicht einer Einteilung in Partner erster und zweiter Klasse gleichkomme, sondern unterschiedliche Entwicklungsverläufe berücksichtigen solle.

Darüber hinaus liefern die Geschehnisse rund um die Östliche Partnerschaft gute Argumente für eine Neufassung der Europäischen Sicherheitsstrategie (ESS). Ein Experte beschrieb die ESS als in hohem Maße überholt: Sie sei 2003 von den damals nur 15 Mitgliedstaaten im Kontext erheblicher Uneinigkeit über den Irak-Krieg verabschiedet worden. In Anbetracht dessen könnte eine ernsthafte Debatte über die latenten Interessensunterschiede der nun 28 Mitglieder vis-à-vis der Östlichen Partnerschaft und Russland auch als ein einheitsstiftender Moment zur Neujustierung der ESS genutzt werden. Denn erst wenn verschiedene Meinungen offen angesprochen werden, lassen sich Kompromisse finden und politische Strategien formulieren. Der damalige Fokus auf den internationalen Terrorismus könnte nunmehr, so ein informierter Beobachter, durch ein verstärktes Augenmerk auf gegenwärtige regionale Herausforderungen in den EU-Nachbarschaften ergänzt werden.
Führungsmöglichkeiten erkennen und ergreifen

Die Verbesserung der Kommunikation und Nachjustierung von Strategien sind enorme Herausforderungen für eine Gemeinschaft von nicht weniger als 28 Staaten. In einem solchen Kontext wird mit wachsender Größe auch die Rolle von Führungsakteuren zunehmend wichtig. Eine Führungsverantwortung soll in diesem Zusammenhang nicht mit unilateralem Durchboxen von Partikularinteressen gleichgesetzt werden, sondern verweist vielmehr auf die Fähigkeit zur Zusammenführung von verschiedenen Interessen für einen allseitig vertretbaren Kompromiss. Insbesondere die nordischen Länder verfügen über eine weit beachtete Expertise in der erfolgreichen Mediation in internationalen Konflikten basierend auf universellen Werten. Zudem können die baltischen Staaten auf einzigartige Erfahrungen verweisen in ihren eigenen demokratischen Transformationsprozessen und den Beziehungen zu Russland. Auf der anderen Seite wurden in letzter Zeit heimische und ausländische Stimmen lauter, die von Deutschland eine größere internationale Verantwortung fordern. Mehrere Redner unterstrichen daher das Potenzial für eine deutsch-nordisch-baltische Partnerschaft, die innerhalb der Europäischen Union eine kollektive Führungsrolle für wichtige Reformen in Bereichen von gemeinsamem Interesse übernehmen könnte.

Ein konkreter institutioneller Rahmen für eine verbesserte regionale Kooperation könnte die Europäische Zentralbank (EZB) sein. Ein Experte wies auf die Tatsache hin, dass mit der Aufnahme Litauens in die Eurozone der EZB-Rat als oberstes Beschlussorgan auf 19 Mitglieder anwächst, sodass nicht mehr jedes Land bei allen Entscheidungen stimmberechtigt sein wird. Bei dem dann geltenden Rotationsprinzip könnte es unter Umständen vorteilhaft sein, wenn sich die nordischen, baltischen und deutschen Repräsentanten konsultieren und ihre Aktionen koordinieren, sollte ein Partner vorübergehend nicht abstimmen dürfen. 
Der Reformprozess der Östlichen Partnerschaft stellt eine weitere potenzielle regionale Kooperationsplattform dar. Mehrere Teilnehmer teilten die kritische Ansicht, dass die Strategie bislang zu sehr technisch-bürokratisch betrieben wurde, ohne den immanenten politischen Implikationen genügend Aufmerksamkeit zu schenken. Trotz oder gerade wegen der folgenschweren Ereignisse in Bezug auf die Ukraine wäre die Europäische Union gut beraten, sich schrittweise vom jetzigen reaktiven Krisenmanagement zu einer proaktiven Strategieausrichtung zu bewegen. Mit ihren historischen Erfahrungen sahen viele Redner die baltischen Staaten gut positioniert, in Kooperation mit nordischen und deutschen Partnern für eine verstärkt strategische und sicherheitspolitische Dimension innerhalb der Östlichen Partnerschaft einzutreten. Solch ein kollektives Bemühen könnte dazu führen, dass die Strategie fortan verstärkt in real-politischen Zusammenhängen gedacht und verankert wird.

$\mathrm{Ob}$ in der östlichen Nachbarschaft oder darüber hinaus, bedarf die Ausgestaltung der Europäischen Union als außenpolitischer Akteur offensichtlich einer aktiven Führung. Ein breites Arsenal an Instrumenten steht formal insbesondere seit dem Inkrafttreten des Vertrags von Lissabon zur Verfügung, jedoch wird dessen Potenzial in den Nachwirkungen der Finanzkrise bislang nicht vollends ausgeschöpft. Diese Diskrepanz führt zum vielfach kritisierten Auseinanderklaffen von Anspruch und Wirklichkeit in der EU-Außenpolitik. Traditionell wurde dieser Politikbereich von den großen Mitgliedstaaten wie Frankreich und Großbritannien vorangetrieben und dominiert. Einfluss leitet sich heutzutage jedoch nicht automatisch aus der Größe eines Landes $\mathrm{ab}$, wie es ein Experte formulierte, sondern kann auch von neuen Ideen und großem Engagement für die gemeinsamen Werte herrüh- ren. Die nordischen und baltischen Länder können demzufolge mit der Unterstützung von Deutschland eine Führungsrolle für außenpolitische Themen übernehmen, in denen sie bereits ausgewiesen sind. Sie sind zum Beispiel exzellent aufgestellt, um innerhalb der Europäischen Union ein ambitionierteres ziviles Krisenmanagement oder verstärkte Mediationskapazitäten fortzuentwickeln.

Während Führungsverantwortung gewöhnlich mit den Verantwortlichen in den Regierungen assoziiert wird, betonte ein Diskussionsteilnehmer das große Potenzial für eine verbesserte Zusammenarbeit zwischen den nationalen Parlamenten. Die Reformen des Vertrags von Lissabon gebe den Legislativen eine Reihe von Möglichkeiten an die Hand, um EU-Entscheidungsprozesse zu beeinflussen. Obschon die Zusammenarbeit zwischen den Parlamenten in der Region in den letzten Jahren intensiviert wurde, sollte sich eine verstärkte deutsch-nordisch-baltische Partnerschaft auch auf dieser Ebene widerspiegeln. Neue legislative Kompetenzen könnten durch eine regionale Kooperation besser ausgefüllt und maximiert werden. Dies würde den Parlamenten helfen, Richtungsentscheidungen aktiv zu gestalten anstatt nur abzusegnen. Außerdem könnte somit die EU-Politik insgesamt auf eine größere demokratische und rechenschaftspflichtige Basis gestellt werden.

Zusammenfassend lässt sich festhalten, dass das DNBF seinem Ruf als offene Gesprächsplattform zu aktuellen Themen gerecht wurde. Entscheidenden Anteil an dem anregenden und kritischen Austausch hat dabei die informelle Atmosphäre. Inhaltlich sorgt die Vielschichtigkeit der deutsch-nordisch-baltischen Zusammenarbeit im Kontext der Europäischen Union sicherlich auch in den nächsten Jahren für genügend Gesprächsstoff. 